\title{
Targeting cancer stem cells expressing an embryonic signature with anti-proteases to decrease their tumor potential
}

\author{
CY Darini ${ }^{1,2,6,7}$, P Martin ${ }^{1,2,6}$, S Azoulay ${ }^{3}$, M-D Drici ${ }^{4}$, P Hofman ${ }^{5}$, S Obba ${ }^{1,2,8}$, C Dani ${ }^{1,2}$ and A Ladoux ${ }^{*, 1,2}$
}

Cancer stem cells (CSCs) are a specific subset of cancer cells that sustain tumor growth and dissemination. They might represent a significant treatment target to reduce malignant progression and prevent tumor recurrence. In solid tumors, several hierarchically organized CSC clones coexist, even within a single tumor. Among them, CSCs displaying an embryonic stem cell 'stemness' signature, based on the expression of Oct-4, Nanog and Sox2, are present in distinct high-grade tumor types associated with poor prognosis. We previously designed a model to isolate pure populations of these CSCs from distinct solid tumors and used it to screen for molecules showing selective toxicity for this type of CSC. Here we show that human immunodeficiency virus (HIV)-protease inhibitors (HIV-PIs) specifically target CSCs expressing an embryonic signature derived from tumors with distinct origins. They reduced proliferation in a dose-dependent manner with a higher specificity as compared with the total population of cancer cells and/or healthy stem cells, and they were efficient in inducing cell death. Lopinavir was the most effective HIV-PI among those tested. It reduced self-renewal and induced apoptosis of CSCs, subsequently impairing in vivo CSC-induced allograft formation. Two key pharmacophores in the LPV structure were also identified. They are responsible for the specificity of CSC targeting and also for the overall antitumoral activity. These results contribute to the identification of molecules presenting selective toxicity for CSCs expressing an embryonic stemness signature. This paves the way to promising therapeutic opportunities for patients suffering from solid cancer tumors of poor prognosis.

Cell Death and Disease (2013) 4, e706; doi:10.1038/cddis.2013.206; published online 4 July 2013

Subject Category: Cancer

Cancer stem cells (CSCs) represent a reservoir of selfsustaining cells with a high malignant tumor formation potential. They efficiently form tumors and remain in patients even following conventional therapy. ${ }^{1}$ These cells are not very affected by therapies designed to eliminate rapidly dividing cells, that is, the major component of solid tumors. If the tumor size is reduced by killing the bulk of tumor cells, CSCs can survive and regenerate new tumors. ${ }^{2}$ Any agent able to distinguish between CSCs, the total cancer cell population and, essentially, normal stem cells may constitute the first step toward elaborating more successful cancer treatments. However, identification of such molecules is hindered by the heterogeneity of CSC populations, even within one tumor type $^{3-5}$ and the availability of pure and well-characterized CSC populations.

Indeed, vast CSC phenotypic diversity has been described in distinct solid tumors based on the expression of cell surface markers such as CD133, CD44, ${ }^{6-9}$ the activity of enzymes such as ALDH or the ability to exclude cytotoxic molecules. ${ }^{10,11}$ Co-expression of any of these markers makes it difficult to isolate a sufficient amount of pure CSC populations for drug screening. Furthermore, CSC purification requires a reliable signature, which is somewhat inconsistent with the fickle behavior of these cells. ${ }^{3,4}$ From a functional standpoint, CSCs are defined by their ability to self-renew, which is essential for their maintenance. This property is also important to indefinitely perpetuate the growth of a malignant cell population ${ }^{12}$ and to recapitulate the hierarchy of the original tumor. ${ }^{5,13}$ Recently, we used a self-renewal gene tracking strategy to isolate pure populations of CSCs upon Oct-4 (pou5f1) expression. These populations also expressed Nanog and Sox2, which are all essential genes for the maintenance of ES cells. ${ }^{14}$ Besides their high tumor potential, CSCs expressing an embryonic stemness signature are able

\footnotetext{
${ }^{1} \mathrm{CNRS}$, iBV, 28 Avenue de Valombrose, F-06107 Nice, France; ${ }^{2}$ University of Nice-SophiaAntipolis, iBV, 28 Avenue de Valombrose, F-06107 Nice, France; ${ }^{3}$ Institut de Chimie de Nice UMR 7272, University of Nice-Sophia Antipolis, Parc Valrose, 06108 Nice, France; ${ }^{4}$ Pharmacology Department, Nice University Medical Center Hospital, 30 Avenue de la Voie Romaine, 06002 Nice, France and ${ }^{5}$ INSERM ERI 21/EA 4319 and Human Biobank, Faculty of Medicine and Pasteur Hospital, CHU of Nice, University of Nice Sophia-Antipolis, 28 Avenue de Valombrose, 06107 Nice, France

${ }^{*}$ Corresponding author: A Ladoux, CNRS, iBV, Institute of Biology Valrose, University of Nice Sophia-Antipolis, 28 Avenue de Valombrose, 06107 Nice Cedex 2, France. Tel: +33 4933777 04; Fax: +33 4933770 58; E-mail: ladoux@unice.fr

${ }^{6}$ These authors contributed equally to this work.

${ }^{7}$ Present address: IRCM, 110 Avenue des Pins Ouest, Montreal H2W 1R7, Canada.

${ }^{8}$ Present address: INSERM U1065, Centre Méditerranéen de Médecine Moléculaire, 151 Route de Saint-Antoine de Ginestière BP2 3194, 06204 Nice cedex 3, France. Keywords: cancer stem cells; HIV-protease inhibitors; stemness signature; apoptosis; Oct-4

Abbreviations: AIDS, acquired immune deficiency syndrome; APV, amprenavir; CASP3, caspase 3; CSC, cancer stem cell; ER stress, endoplasmic reticulum stress; ES, embryonic stem; FITC, fluorescein isothiocyanate; HAART, highly active anti-retroviral therapy; HIV, human immunodeficiency virus; IDV, indinavir; LPV, lopinavir; NFV, nelfinavir; PARP, poly (ADP-ribose) polymerase; PIs, protease inhibitors; RTV, ritonavir; SAR, structure-activity relationship; SQV, saquinavir

Received 07.3.13; revised 05.5.13; accepted 14.5.13; Edited by Y Shi
} 
to form metastatic tumors mainly in the lungs. This 'stemness' signature is also found in human tumors associated with tumor dissemination, poor prognosis ${ }^{15-17}$ and hard to control disease processes. ${ }^{2}$ Like other CSC types, these CSCs are insensitive to most cancer treatments, including chemo- or radiation therapy. ${ }^{18-23}$ They represent one of several important CSC populations that could be targeted to reduce malignant progression, dissemination and consequently improve the patients' outcome.

Approaches that rely on an alteration of CSC properties are of interest to eliminate this population. As CSCs display an unlimited self-renewal capability governed by proper gene networks, any alteration in their expression may impede tumor development. In our model, disruption of Oct-4 expression after knockdown using RNA interference impairs self-renewal and is detrimental to both tumor and metastasis developments. ${ }^{14}$ This approach is of great interest but several factors hamper its use in vivo. For instance, degradation of small RNAs by enzymes is responsible for poor penetration into tissues. ${ }^{24}$ Targeting CSCs with specific monoclonal antibodies to surface markers such as CD44 was proposed as a powerful approach to treat leukemia ${ }^{25}$ and possibly breast cancer. ${ }^{26}$ Unfortunately, eradication using specific monoclonal antibodies to surface markers was inappropriate as no common cell surface markers have been identified in Oct-4expressing CSCs. ${ }^{14}$

These limitations prompted us to screen for molecules that could selectively kill CSCs expressing an embryonic signature and enabled us to identify a well-defined class of protease inhibitors (PIs) as candidates.

Human immunodeficiency virus (HIV)-PIs are of paramount importance for highly active anti-retroviral therapy against HIV (highly active anti-retroviral therapy, HAART). HAART was found to clearly improve the quality of life of acquired immune deficiency syndrome (AIDS) patients by lowering the viral charge and increasing the number of CD4-positive $T$ cells, thus contributing to the restoration of the patients' immune system. ${ }^{27,28}$ In addition to their anti-viral benefits, the efficacy of HIV-PIs to decrease AIDS-associated Kaposi sarcoma ${ }^{29,30}$ has raised interest in their distinct antitumor properties. They have been shown to efficiently reduce the tumor mass of aggressive neoplasms such as glioblastomas or ovarian cancers, ${ }^{31,32}$ to reduce xenograft formation from prostatic tumors $^{33}$ as well as the growth of hepatocarcinomas in vivo. ${ }^{34}$ However, these studies were performed indiscriminately on total cancer cell populations, thus hindering any identification of PI-specific effects on CSCs, including those expressing an embryonic signature.

In this study, we show that among HIV-PIs, lopinavir (LPV), one of the most widely used HIV-PIs, efficiently distinguishes CSCs among cancer cells and selectively reduces proliferation and self-renewal of Oct-4-expressing CSCs isolated from different types of solid tumor. ${ }^{14}$ Hence, both the total population of cancer cells from the same tumor and healthy stem cells are only affected at higher drug concentrations. Structure-activity relationship (SAR) experiments performed using key intermediates for LPV synthesis led to the identification of essential pharmacophores for LPV-antitumor specificity and activity. LPV-induced death of CSCs was accompanied by activated-caspase 3 (CASP3) expression and cleavage of the DNA repair enzyme poly (ADP-ribose) polymerase, that is, PARP-1, which represents a hallmark of apoptosis. ${ }^{35}$ Finally, in vivo treatment of mice with a fixed association of LPV and ritonavir (RTV) resulted in a reduction in allograft formation, indicating a beneficial effect on tumor regression.

Overall, these results indicate that HIV-PIs selectively and potently kill CSCs bearing a high malignant potential and an embryonic stemness signature. This represents a novel and promising approach to directly target this type of cells responsible for tumor growth and cancer relapse.

\section{Results}

HIV-PIs preferentially decrease CSC proliferation. Proliferation of CSCs and the total tumor cell population was measured in the presence of salinomycin, a potassium ionophore reported to specifically affect breast cancer CSCs, ${ }^{36}$ and of different PIs.

Salinomycin reduced proliferation of both CSCs and total population of the same parental tumor with a comparable potency (Figure1a). The range of concentrations corresponds to that reported to efficiently kill breast CSCs. This indicated that salinomycin did not preferentially target CSCs expressing an embryonic signature.

In contrast, among the Pls tested, we found that nelfinavir (NFV), saquinavir (SQV) and RTV were more efficient in reducing $\mathrm{CSC}$ growth. The $\mathrm{IC}_{50} \mathrm{~S}$ for proliferation inhibition were: 2, 3 and $3.5 \mu \mathrm{M}$, respectively, (Figures $1 \mathrm{~b}-\mathrm{d}$ ).

Amprenavir (APV) and indinavir (IDV) decreased proliferation of both the total and CSC populations with no selectivity and similar efficacy ( $\mathrm{IC}_{50}$ in the $10 \mu \mathrm{M}$ range; data not shown).

LPV stood out from all PIs and was the most efficient PI affecting the growth of CSCs isolated from two independent tumors $\left(0.6<\mathrm{IC}_{50}<1.5 \mu \mathrm{M}\right.$; Figures $2 \mathrm{a}$ and $\left.\mathrm{b}\right)$. The $\mathrm{IC}_{50} \mathrm{~S}$ for proliferation inhibition were lower than the therapeutic range of LPV for patients under HAART therapy $(2-12 \mu \mathrm{g} / \mathrm{ml}$, i.e., $3.2-19 \mu \mathrm{M})$. For the highest concentrations tested, LPV not only reduced growth but also affected CSC viability as only a few cells remained attached. Depending on the organ from which the tumor was derived, LPV was 25-75 times more efficient in reducing CSC proliferation as compared with the total tumor population $\left(25 \mu \mathrm{M}<\mathrm{IC}_{50}<50 \mu \mathrm{M}\right)$ (Figures 2a and b). Similar results were obtained with healthy mesenchymal stem cells, as LPV impaired their proliferation with $\mathrm{IC}_{50}>50 \mu \mathrm{M}$ (Figure 3). In addition, LPV also dose-dependently reduced the clonogenic potential of Oct-4-expressing CSCs, as measured by the percentage of cells able to form colonies on methylcellulose (Figure 2c). This indicated that the decreased proliferation was accompanied by a decreased ability to grow at a single cell level, thus suggesting a loss of self-renewal capacity.

By order of selectivity, Pls can be ranked from the most to the least potent as follows: $L P V>N F V>S Q V \geq R T V>$ $\mathrm{APV}=\mathrm{IDV}$.

Structure-activity involved in LPV selectivity. As a preliminary SAR study, key intermediates for LPV synthesis (Figure 3a) were prepared and assessed in proliferation assays. 

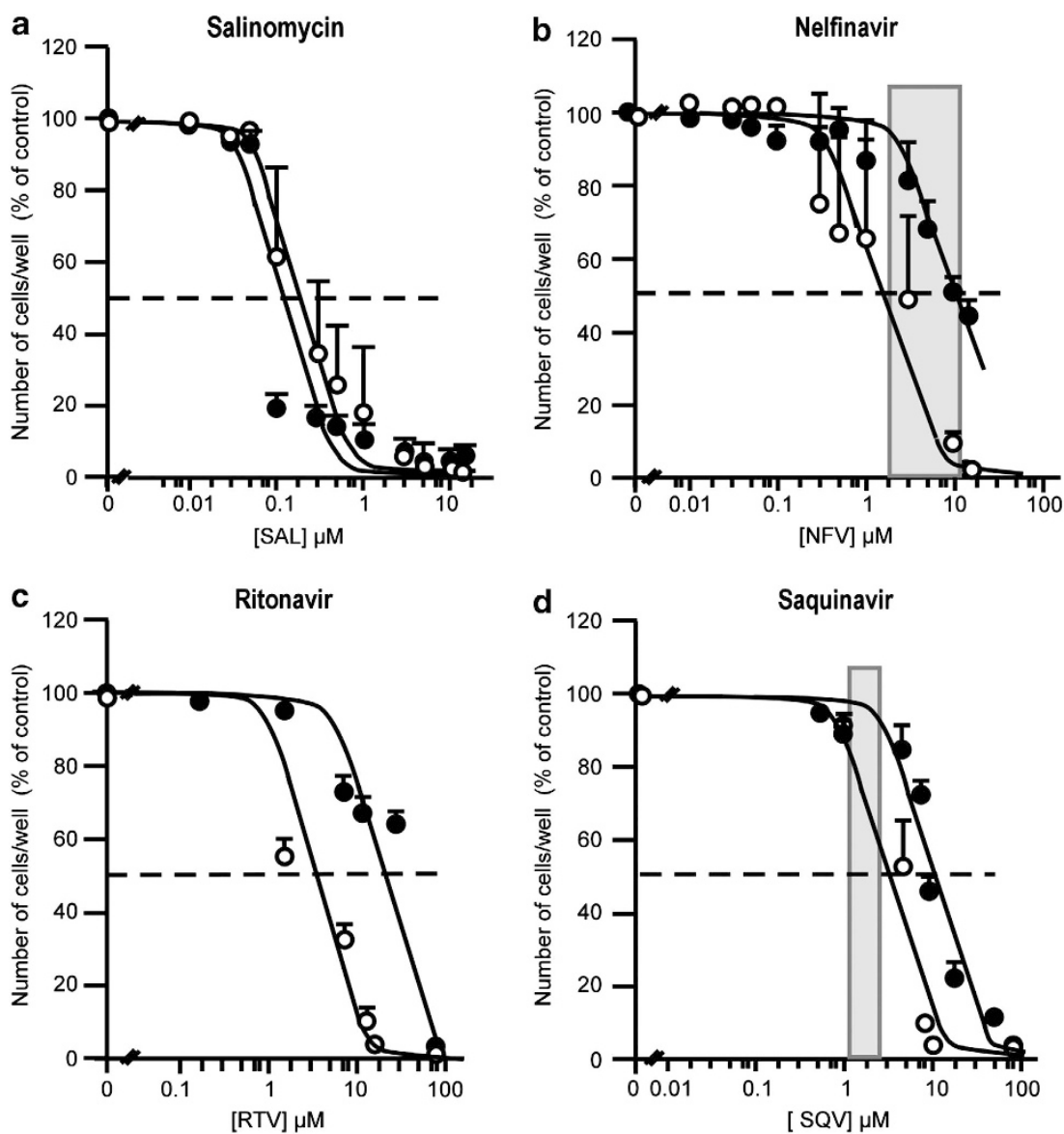

Figure 1 PIs selectively decrease the proliferation of CSCs compared with the total tumor population while salinomycin is efficient on both populations. Dose-response curves for the PI-induced inhibition of cell proliferation for CSCs (open circle) or the total tumor population (closed circles) from an adenocarcinoma in response to the potassium ionophore salinomycin (a) and to NFV (b), RTV (c), SQV (d). Grey zones represent the plasma concentrations of the corresponding PI in treated patients, as reported in the literature. The results represent the mean \pm S.E.M. of three experiments carried out in triplicate. Error bars were omitted when the S.E.M. was smaller than the size of the symbol. $\mathrm{IC}_{50} \mathrm{~S}$ were calculated from the curves

When CSCs were grown in the presence of increasing concentrations of (2S)- $N$-[(2S,4S,5S)-5-amino-4-hydroxy1,6-diphenylhexan-2-yl]-3-methyl-2-(2-oxo-1,3-diazinan-1-yl) butanamide (or LPV-precursor) P-1, no toxic effect was observed on CSCs at LPV concentrations equivalent to or higher than those required to kill the cells (Figures $3 \mathrm{~b}$ and $\mathrm{c}$ ). This indicated that the dimethylphenoxyacetic acid moiety was essential for the antitumoral properties of LPV.

In contrast, $N$-[(1S,2S,4S)-4-amino-2-hydroxy-5-phenyl-1(phenylmethyl)pentyl]-2-(2,6-dimethylphenoxy) acetamide (or LPV-precursor) P-2, an intermediate containing the previously identified pharmacophore but without the L-valine carboxylic acid derivative part, displayed efficacy in reducing CSC proliferation in a similar range as compared with LPV. This molecule was also more efficient than LPV in killing the total cancer cell population (Figure 3d), indicating that this chemical group is crucial to distinguish between the two cancer cell populations. Indeed, loss of this pharmacophore induces a high toxicity in human mesenchymal stem cells as compared with LPV, with an $\mathrm{IC}_{50}$ of $4-5 \mu \mathrm{M}$ (Figure 3e).

We then used a modified LPV molecule, referred to as P-3, where the central hydroxyl group responsible for anti-protease activity was protected. When CSCs were grown in the presence of increasing P-3 concentrations, a toxic effect was observed at concentrations higher than those required for LPV $\left(\mathrm{IC}_{50}=6 \mu \mathrm{M}\right)$, indicating that the anti-protease activity was essential for the anti-tumor property of LPV (Figures 3b and c). However, P-3 presented a very low (even no) toxicity toward the total cancer cell population $\left(\mathrm{IC}_{50}>50 \mu \mathrm{M}\right.$, Figures $3 \mathrm{~b}-\mathrm{d}$ ), indicating that the anti-protease activity was greater for toxicity than for selectivity. Moreover, P-3 did not show high toxicity as compared with LPV on mesenchymal stem cells (Figure 3e).

Overall, these results indicate that the anti-proliferative properties of LPV were associated with the presence of the dimethylphenoxyacetic acid moiety and the functional hydro$x y l$ group that is essential for the anti-protease activity, while a distinct part of the molecule was able to selectively target CSCs.

LPV induces apoptosis in CSCs. Oct-4-expressing CSCs or cells from the total cancer cell population were treated with individual PIs for $48 \mathrm{~h}$ and apoptosis induction was assessed by the loss of plasma membrane asymmetry, as measured 

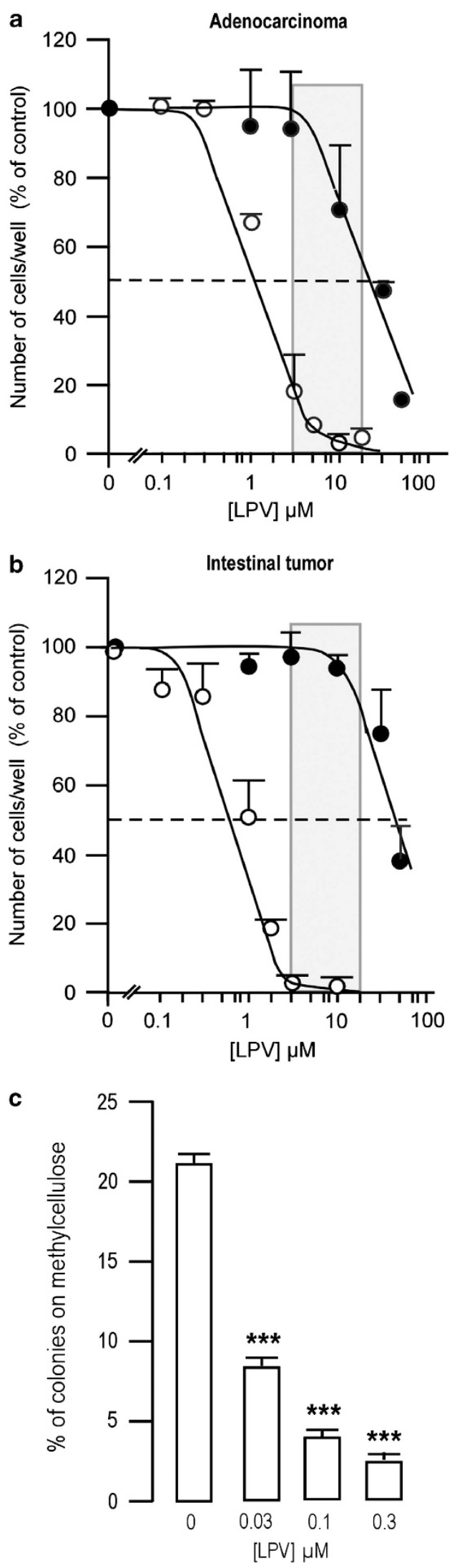

by annexin-V-labeling using flow cytometry. A 48-h treatment with LPV $(1 \mu \mathrm{g} / \mathrm{ml}$, i.e., $1.6 \mu \mathrm{M})$ induced an increase in annexin-V-fluorescein isothiocyanate (FITC) labeling in $77.5 \pm 12.4 \%(n=4)$ of the cells, while a similar treatment did not induce significant labeling in the total cancer cell population (Figure 4a). In this latter population, 48-h treatment with $20 \mu \mathrm{g} / \mathrm{ml}$ (i.e., $32 \mu \mathrm{M}$ ) LPV was required to significantly increase the percentage of labeled cells to $31 \pm 6 \%(n=3)$ as compared with non-treated cells (data not shown). CSC treatment with NFV $(1-3 \mu \mathrm{M})$ increased the percentage of annexin-V-FITC-positive cells to $69.2 \pm 4.7 \%$ and $78.0 \pm 5.4 \%$, respectively $(n=4)$, whereas higher RTV concentrations ( $10 \mu \mathrm{g} / \mathrm{ml}$, i.e., $14 \mu \mathrm{M}$ ) were required to obtain an equivalent result $(74.2 \pm 10.2 \%$ of labeled cells $(n=6)$; Figure 4a).

Comparable results were obtained with Oct-4-expressing CSCs derived from another tumor (Supplementary Figure 1A). These results indicate that apoptosis, via annexin- $V$ labeling, could be detected before a decrease in cell proliferation.

Regardless of the CSCs used, this process was associated with CASP3 activation (Figures $4 \mathrm{~b}$ and $\mathrm{c}$ and Supplementary Figures $1 \mathrm{~B}$ and $\mathrm{C}$ ) and with PARP-1 cleavage (Figure $4 \mathrm{~d}$ and Supplementary Figure 1D). Cleaved-CASP3 was detected in cells treated with LPV for $24 \mathrm{~h}$ at $1-3 \mu \mathrm{g} / \mathrm{ml}$ concentrations. Its expression increased significantly 10- to 14-fold depending on the cell line, in a dose-dependent manner (Figure 4c and Supplementary Figure 1C). In parallel, LPV treatment induced PARP-1 cleavage in a dose-dependent manner, as measured by the specific accumulation of an $89 \mathrm{kDa}$ fragment in cells (Figure $4 \mathrm{~d}$ and Supplementary Figure 1D). LPV thus enhanced annexin- $V$ labeling, increased expressions of the activated form of CASP3 and cleaved form of PARP-1 before reducing cell proliferation and altering cell viability.

We next checked if endoplasmic reticulum (ER) stress was involved in this process. We did not note any significantly increased expression of the $78 \mathrm{kDa}$ glucose-regulated protein (also called BiP) after treatment with $5 \mu \mathrm{g} / \mathrm{ml} \mathrm{LPV}$, that is, a concentration able to induce CASP3 cleavage within $24 \mathrm{~h}$ (Supplementary Figure 2). Moreover, we did not observe any activation of the pathways downstream of BiP (such as eukaryotic initiation factor 2 phosphorylation or active X-box binding protein 1 synthesis) (data not shown). LPV-induced apoptosis in CSCs thus likely was related to activation of the caspase pathway independently from the activation of ER stress pathways, as reported for other cancer cells. ${ }^{32}$

LPV reduces CSC-induced tumor progression. We next examined the effects of fixed doses of LPV/RTV (4/1 ratio)

Figure 2 LPV decreases the proliferation and clonogenicity of CSCs. Doseresponse curves for LPV-induced inhibition of cell proliferation for CSCs (open circle) or the total tumor population (closed circle) from an adenocarcinoma (a), or an intestinal tumor (b). Grey zones represent the plasma concentrations of LPV in treated patients, as reported in the literature. The results represent the mean \pm S.E.M. of three experiments carried out in duplicate. Error bars were omitted when the S.E.M. was smaller than the size of the symbol. $I_{50} S$ were calculated from the curves. (c) LPV reduces CSC clonogenicity. After growing 170 cells on methylcellulose for 3 weeks, the percentage of colonies obtained from Oct-4-expressing cells was calculated. Mean \pm S.E.M. was representative of three independent experiments $\left.{ }^{* * *} P<0.001\right)$ 
administrated in mice, with RTV used to stabilize LPV in vivo. This ratio was chosen according to the standard prescription given to treat AIDS patients. We checked that the presence

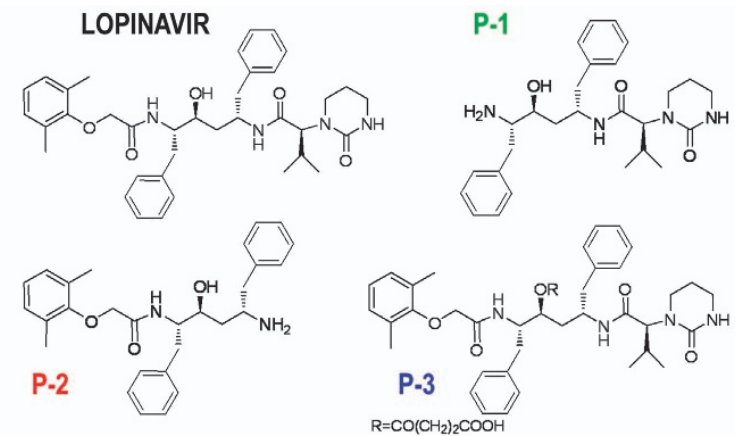

b

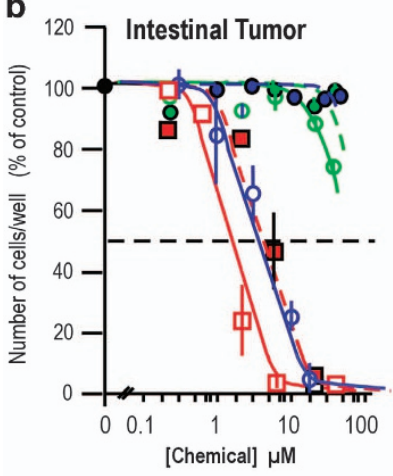

d

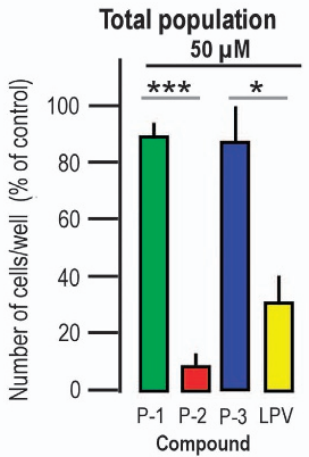

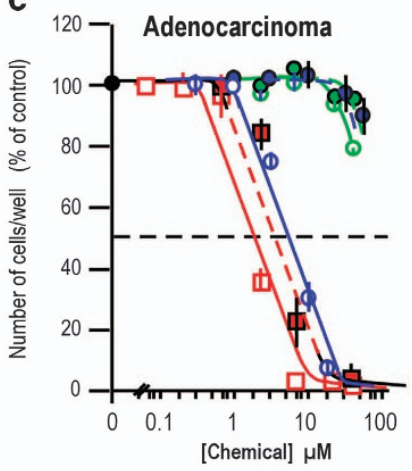

e

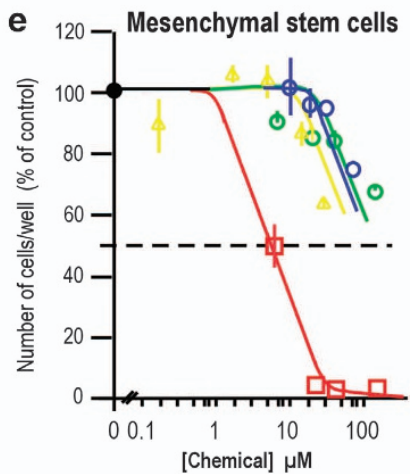

Figure 3 Structure activity of LPV for its antitumoral properties. (a) Structures of LPV and pharmacophores. (b) Dose-response curves for pharmacophore P-1 (green circles), pharmacophore P-2 (red square) or pharmacophore P-3 (blue circle) -induced inhibition of cell proliferation for CSC (open symbols) or the total population (filled symbols and dashed curves) of an intestinal tumor. The results represent the mean \pm S.E.M. of three experiments carried out in duplicate. (c) Dose-response curves for pharmacophore P-1 (green circles), pharmacophore P-2 (red square) or pharmacophore P-3 (blue circle) -induced inhibition of cell proliferation for CSC (open symbols) or the total population (filled symbols and dashed curves) of an adenocarcinoma. The results represent the mean \pm S.E.M. of three experiments carried out in duplicate. (d) Comparison between the effects of LPV and pharmacophores P-1, P-2, P-3 on cell proliferation of the total population of an intestinal tumor. The results represent the mean \pm S.E.M. of three experiments carried out in duplicate $\left({ }^{*} P<0.05 ;{ }^{* \star} P<0.001\right)$. (e) Dose-response curves for LPV (yellow symbol and curve), pharmacophore P-1 (green circle and curve), or pharmacophore P-2 (red square and curve), or pharmacophore P-3 (blue circle and curve) -induced inhibition of cell proliferation for human mesenchymal stem cells. The results represent the mean \pm S.E.M. of three experiments carried out in duplicate. For panels $(\mathbf{b}-\mathbf{e})$, error bars were omitted when the S.E.M. was smaller than the size of the symbol of RTV did not significantly change the $\mathrm{IC}_{50}$ for LPV-induced inhibition of proliferation (data not shown). Murine clearance of LPV/RTV was substantial as little if any LPV could be detected in the mouse serum 8-h post-administration. ${ }^{37}$ This prompted us to administer the drugs twice daily, with a minimum interval of $8 \mathrm{~h}$. The LPV/RTV dosage had to be in the range of prescriptions established for humans and appropriate for a long-term treatment of mice. The chosen LPV dose was thus below that recapitulating the drug impregnation similar to the pattern observed in humans ${ }^{37}$ (i.e., causing unwanted secondary effects).

After 7 days of treatment, no significant difference between mice receiving placebo or active treatment was noted for tumor engraftment (Figure 5Aa). Tumor growth began to decrease slightly after 20-30 days of treatment depending on the parental tumor origin, with CSCs derived from adenocarcinomas being more sensitive to the treatment (Figure 5Ab). After 55 days of treatment, allograft development was significantly reduced as compared with the results in mice receiving placebo, irrespective of the parental tumor the CSCs derived from (Figures $5 \mathrm{Ac}$ and $\mathrm{Bc}$ ).

Histological analysis of the allograft sections confirmed these results. Figure 5B (panels $d$ and e) shows that in mice receiving placebo, allografts developed and presented an undifferentiated phenotype, while the treatment actively restrained the efficacy of CSCs to proliferate and form undifferentiated allografts.

\section{Discussion}

Cancer cell populations are organized in a CSC-oriented hierarchy. ${ }^{13}$ They are of paramount importance for tumor development because they tend to disseminate and form metastases. Conventional therapies are efficient in significantly reducing the tumor burden by eliminating the bulk of cancer cells. Hence, new therapies targeting the CSCs are of interest in that they could purge tumors of the highly malignant CSCs population.

Among the multiple types of CSCs that have been identified in distinct solid tumors, ${ }^{38}$ Oct-4-positive CSCs are associated with high-grade and poor prognosis tumors. ${ }^{15-17}$ We have developed and previously described ${ }^{14}$ a model to study pure populations of these CSCs from different tumor origins. This model was used to screen for drugs able to specifically kill these cells as compared with the total tumor cell population or to healthy stem cells. HIV-PIs were found to be an efficient antitumor therapeutic class because some of them reduced proliferation, clonogenicity and selectively induced cell death in the CSC population, thus restraining CSC-induced allograft formation.

HIV-PIs are administrated to HIV-positive patients as part of HAART. ${ }^{28}$ They are peptidomimetic drugs designed to mimic the peptide bond targeted by the viral protease but not by any other mammalian endopeptidase, ${ }^{39}$ which means they have a good specificity of action with tolerable adverse effects. HAART has been a major step in the management of HIV infection as it has extended patients' lives by both reducing the viral charge and reconstructing the naive and memory T-cell repertoires, thus delaying or reversing the onset of AIDS. ${ }^{27}$ 


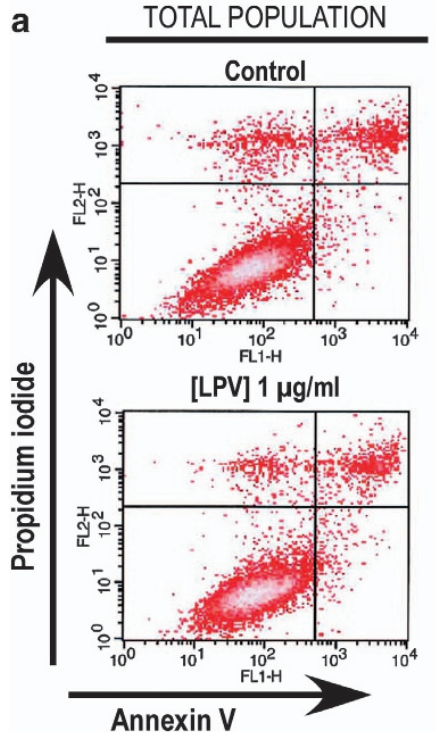

a

C

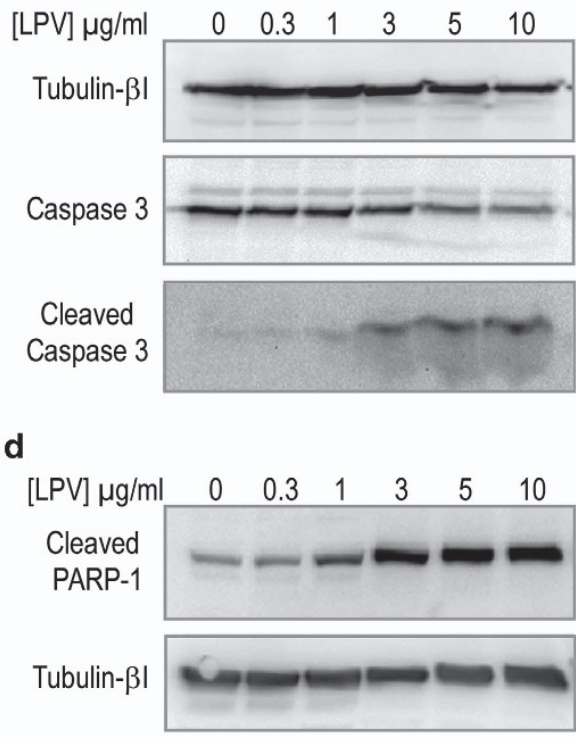

CANCER STEM CELLS
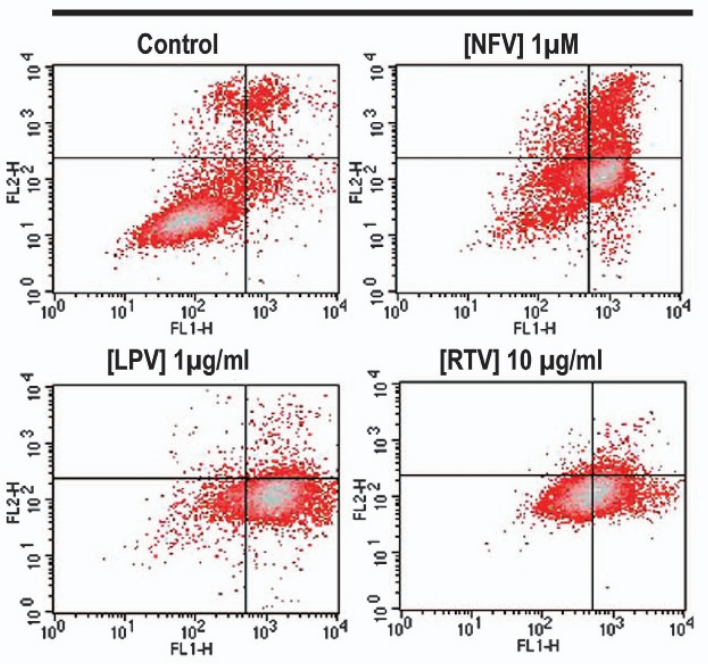

$55 \mathrm{kDa}$

$35 \mathrm{kDa}$

17 kDa

$89 \mathrm{kDa}$

$55 \mathrm{kDa}$ b
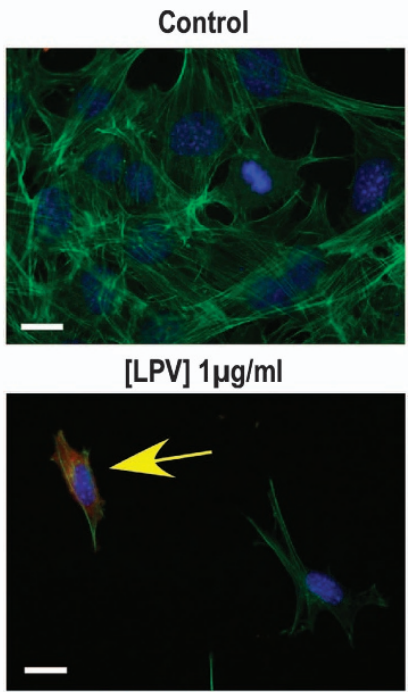

Actin/Activated Caspase 3/ DAPI

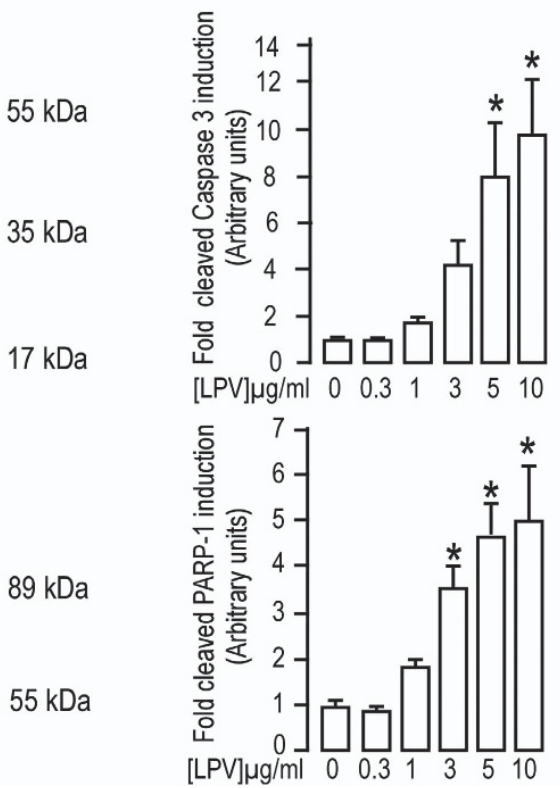

Figure 4 LPV-induced apoptosis in CSCs derived from an adenocarcinoma. (a) Total populations of tumor cells or CSCs were incubated with different Pls for $48 \mathrm{~h}$, as indicated and the apoptotic cells were evaluated by flow cytometry with PI/annexin-V double staining. DMSO was used as solvent and as a negative control. The findings of three (total population) or four (CSCs) independent experiments provided confirmations. (b) CSCs were exposed to LPV and CASP3 activation was determined $24 \mathrm{~h}$ after LPV addition through immunocytochemistry with antibodies against cleaved active CASP3 (17 kDa). Representative images illustrate active CASP3staining (red) in cells following LPV treatment, but cellular red staining is almost absent in cells that did not receive LPV. Phalloidin coupled to Alexa Fluor 488 was used to visualize all cells by means of actin fiber staining (green). Nuclei were labeled blue using DAPI (scale bar, $100 \mu \mathrm{m}$ ). The images are representative of two independent experiments. (c) Western blot analysis of CASP3 cleavage in CSCs treated for $24 \mathrm{~h}$ with vehicle or increasing LPV concentrations. $\beta$-Tubulin I is shown as a loading control. These blots are representative of four independent experiments. The histograms represent the expression of cleaved CASP3 normalized to the tubulin signal. Control condition corresponds to cells that did not receive LPV treatment. Mean \pm S.E.M. obtained from four independent experiments are shown $\left({ }^{\star} P<0.05\right)$. (d) Western blot analysis of PARP-1 cleavage in CSCs treated for $24 \mathrm{~h}$ with vehicle or increasing concentrations of LPV. $\beta$-Tubulin I is shown as a loading control. These blots are representative of four independent experiments. The histograms represent the expression of the $89 \mathrm{kDa}$ fragment resulting from PARP-1 cleavage normalized to the tubulin signal. The control condition corresponds to cells that did not receive LPV treatment. Mean \pm S.E.M. obtained from four independent experiments are shown $\left({ }^{*} P<0.05\right)$

The benefits of HAART were soon noted in AIDS treatment, but also to reduce HIV-associated cancer risk and tumor burden in HIV-infected persons. AIDS patients are more prone to develop certain types of cancers and the antitumoral effect of Pls was first attributed to their efficacy against viruses involved in AIDS-associated malignancies such as Epstein-Barr or Kaposi sarcoma-associated herpes virus. ${ }^{39}$ However, the antitumoral properties of HAART cannot be entirely explained by these effects or by the recovery of normal immune functions. For instance, complete remission 
A $\quad$ D7
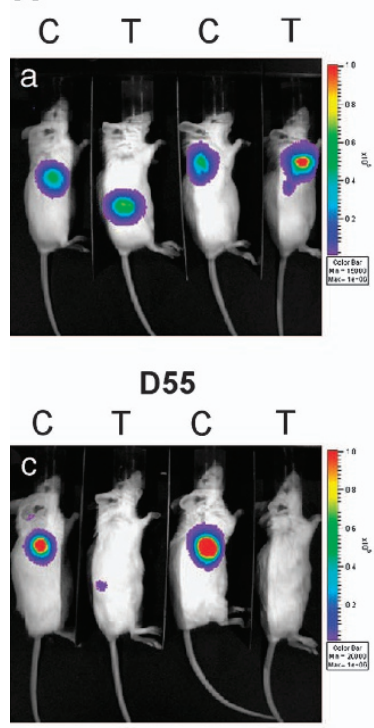

B

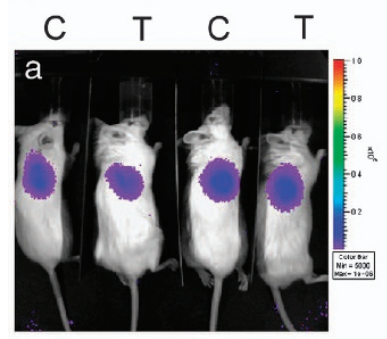

D34

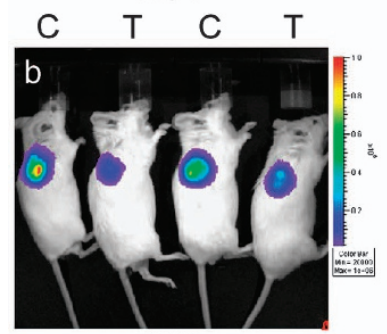

D55

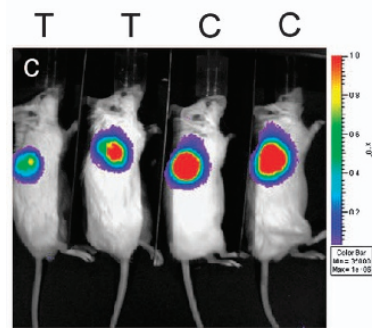

D21
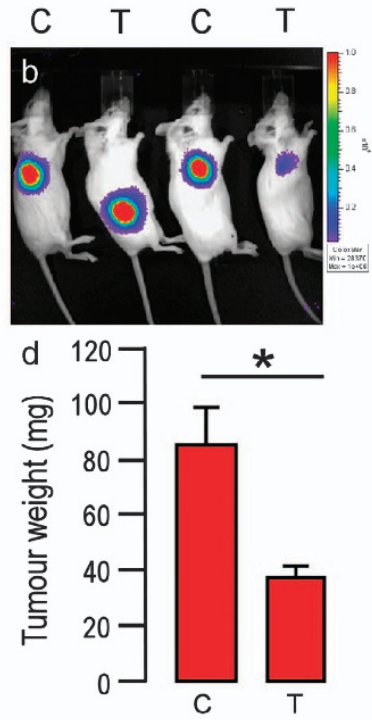

Placebo

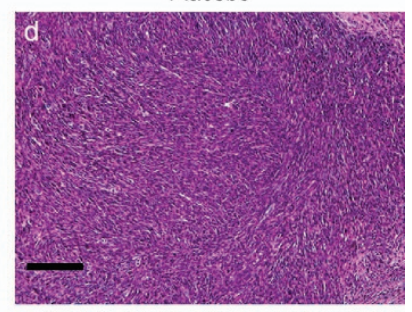

LPV+RTV twice daily
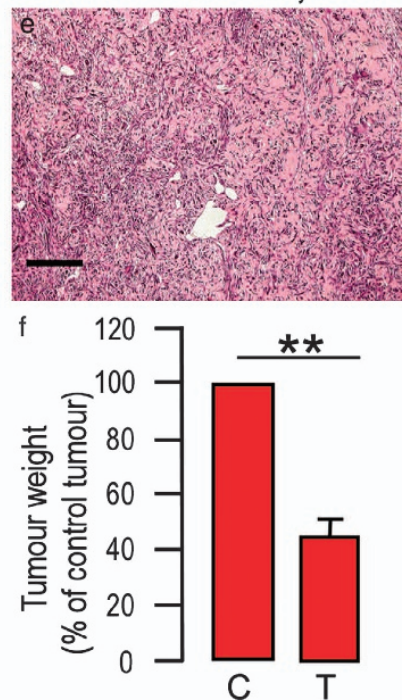

Figure 5 Fixed association of LPV/RTV decreases CSCs-induced allograft formation. CB17/SCID mice transplanted with 250000 cells from an adenocarcinoma $(\mathbf{A})$ or an intestinal tumor $(\mathbf{B})$ were treated twice daily with placebo or LPV/ RTV. In vivo bioluminescent imaging of light emitted by cells reveals a decrease in the size of sites for light emission in mice receiving LPV/RTV after 21 days (Panel $A, b$ ) or 34 days (Panel $B, b)$ of treatment, and this was more pronounced after 55 days of treatment. Tumor weight was assessed after 55 days of treatment and was significantly lower in mice receiving LPV/RTV as compared with those receiving placebo (panel A, d; panel B, f) $\left(n=5,{ }^{*} P<0.05\right.$; $\left.{ }^{\star \star} P<0.01\right)$. Histological analysis of the allografts shown in panel $\mathbf{B}(\mathrm{d}-\mathrm{e})$ confirms that LPV/RTV impaired cell proliferation and allograft formation (scale bar, $100 \mu \mathrm{m}$ )

of Kaposi's sarcoma was more prevalent in patients treated with PIs than in those treated with HIV nucleoside reverse transcriptase inhibitors, ${ }^{29}$ which suggests that they have their own specific antitumoral properties. Furthermore, IDV treatment of Kaposi's sarcoma in HIV-negative patients reduced basic fibroblast growth factor production and lowered the number of endothelial cells, hence lowering the tumor progression and improving the clinical course..$^{30}$

The antitumoral effects of HIV-PIs have been explored in various models. They reduce the tumor mass of aggressive neoplasms such as glioblastomas or ovarian cancers. ${ }^{31,32}$ NFV reduces xenograft formation from myelomas ${ }^{31,40}$ and prostatic tumors. ${ }^{33}$ IDV reduces hepatocarcinoma growth in vivo. ${ }^{34}$ These effects are accompanied by the induction of ER stress, which may in turn trigger cell apoptosis. ${ }^{32,41}$

However, the antitumoral effects of Pls documented in the literature were obtained in total tumor populations and any extrapolation to pure CSC populations would be risky. In close agreement with the previously reported results, we found that NFV was potent in killing Oct-4-expressing CSCs, but the most potent HIV-PI targeting these CSCs was found to be LPV.

LPV was a weak inducer of cell death in the total cancer cell population at concentrations overlapping those efficient for reducing proliferation. This observation is consistent with the effects of LPVs on meningioma cells as they block proliferation through cell cycle inhibition in an AKT-independent manner without induction of apoptosis. ${ }^{42}$ Conversely, our results showed that LPV induced apoptosis efficiently in Oct-4-positive CSCs, as measured by CASP3 activation and PARP-1 cleavage. We did not notice significant ER stress induction in Oct-4-positive CSCs within the range of concentrations inducing apoptosis, indicating that other mechanisms than ER stress might be involved, as previously reported. ${ }^{42}$

This effect was independent of the parental tumor, but relied much more on the CSC type, as equivalent responses were obtained in Oct-4-expressing CSCs derived from an adenocarcinoma or intestinal tumor. In contrast, salinomycin, which preferentially targets breast cancer $\mathrm{CSCs},{ }^{36}$ reduced the proliferation of both Oct-4-positive CSCs and the total tumor population with comparable potency and efficacy. This indicated that, although active, this molecule did not distinguish between the two cancer cell populations. Our results, along with those reported previously, ${ }^{43}$ confirmed the high potential of this molecule for targeting CSCs independently of an embryonic signature expression. Overall, these observations strongly suggest that efficient targeting of CSCs will require molecules specific to the type of CSCs involved.

However, intracellular pathways leading to the collapse of distinct CSC populations upon drug interaction need to be identified in order to be able to develop appropriate treatments. The mechanism by which salinomycin induces specific breast cancer CSC toxicity ${ }^{36}$ remains unclear. Although being efficient in reducing allografts derived from Oct-4-expressing CSCs, there is no evidence yet that oct-4 or nanog or any other genes contributing to expression of the embryonic signature are potential direct targets for LPV. Other possible targets might be genes whose expressions are regulated by this signature.

The SAR study revealed that the anti-protease activity may be involved in the antitumor activity of LPV. LPV inhibits the 
HIV protease, that is, a distinct aspartic protease. This enzyme family occurs in higher vertebrates and has been the focus of enormous interest because of the significant roles of these enzymes in human diseases such as hypertension and Alzheimer's disease. ${ }^{44}$ Among them, cathepsin D is highly expressed in cancer cells and associated with metastasis progression. ${ }^{45}$ LPV has been described to exert its antiviral activity with an $\mathrm{EC}_{50}$ of $0.1 \mu \mathrm{M}^{46}$ and to have a higher ( $>10^{5}$-fold) specificity for HIV protease as compared with the mammalian aspartic proteinases renin, cathepsin $D$ and cathepsin E. ${ }^{47}$ It is therefore unlikely that the antitumoral activity of LPV results from an interaction with cathepsin $D$ as the $\mathrm{IC}_{50}$ measured here was in the $1 \mu \mathrm{M}$ range (i.e., a lower concentration than that efficient to inhibit mammalian aspartic proteases). Furthermore, we did not note any preferential expression of cathepsin D in CSCs (data not shown). Hence, these types of protease appear to be poor candidates for mediating LPV antitumoral properties, albeit the putative inhibition of a yet to be identified aspartic protease cannot be ruled out. ${ }^{39}$ The decrease in anti-protease activity did not modify the selectivity of LPV toward CSCs. Unfortunately, identification of two pharmacophores of the LPV structure crucial for antitumor potency and selectivity did not allow characterization of an intracellular-specific cascade.

The direct intracellular target(s) of LPV and other HIV-PIs in CSCs, cancer cells and healthy stem cells remain to be determined. Several unwanted side effects have been observed in patients under HAART, including lipodystrophy, insulin resistance and, consequently, diabetes, ${ }^{48,49}$ yet no single intracellular cascade has been described. Although HIV-PIs inhibit differentiation of normal mesenchymal stem cells, ${ }^{50}$ they also alter the mitochondrial function in different cell types, ${ }^{51,52}$ indicating that several pathways may be involved, for example, different yet to be identified intracellular pathways involved in LPV antitumor activity. In this regard, the preliminary SAR study generated useful information for designing selective and more potent CSC inhibitors.

Since they are heterogeneous and unstable, new distinct therapies are needed to target the different types of CSC. They would have the advantage of providing personalized cancer treatments that account for both the genetic alterations and CSC status within a tumor, whereas protecting healthy tissues and cells. Overall, our results highlighted that LPV and its derived molecules are promising candidates to selectively reduce the progression of tumors driven by CSCs expressing an embryonic stemness signature. This study represents the first step in the identification of the intrinsic antitumoral properties of HIV-PIs on CSCs. Further studies are needed to decipher the molecular mechanisms underlying HIV-PI antitumoral properties and to design more efficient molecules to wipe out this CSCs population.

\footnotetext{
Materials and Methods

Reagents. Unless specified, all reagents were obtained from Sigma (SaintQuentin Fallavier, France).

Tissue culture media were obtained from LONZA (Levallois-Perret, France) and fetal calf serum from Dutscher SA (Brumath, France). Pls were obtained by extraction from commercially available tablets and capsules. Their purity was assessed by ${ }^{1} \mathrm{H}$ and ${ }^{13} \mathrm{C}$ nuclear magnetic resonance and mass spectroscopy. The key intermediates used for SAR studies were synthesized according to a previously reported procedure. ${ }^{53}$
}

Mouse model and cell culture. CSCs expressing Oct- 4 were isolated from murine tumors that developed in $p 53-/-$ mice expressing GFP and a puromycin resistance gene under the direction of regulatory sequences of the mouse Oct-4 gene, as previously reported. ${ }^{14}$ Briefly, CSCs isolated from an adenocarcinoma and an intestinal tumor, were used in this study. Tumor cells were obtained by gentle mechanical dissociation after digestion in the presence of collagenase $(0.4 \mathrm{mg} / \mathrm{ml}$ Roche Diagnostics, Meylan, France). They were grown under the conditions reported for growing murine ES cells ${ }^{54}$ at $37^{\circ} \mathrm{C}$ in a humid atmosphere in the presence of $5 \% \mathrm{CO}_{2}$. They were isolated after selection with puromycin $(1 \mu \mathrm{g} / \mathrm{ml})$. The cultures were split at the cell growth log phase to prevent overpopulation-induced cell death, and the cells were maintained as previously described.$^{14}$ These CSCs displayed similar properties although they were obtained from two different types of tumor. They expressed other embryonic genes such as Nanog and Sox2, and their tumor potential was driven by Oct-4 expression. ${ }^{14}$

For proliferation experiments, cells were seeded at 60000 cells per well in 12-well plates. After 24-h incubation, HIV-PIs were added to the wells and cells were further incubated for $72 \mathrm{~h}$.

Human mesenchymal stem cells were maintained as previously described. ${ }^{55}$ They were seeded at 30000 cells per well in 12-well plates and treated with LPV or pharmacophores for $72 \mathrm{~h}$.

Clonogenic assay. CSCs mixed in 5/6 Methocult GF 3434 (STEMCELL Technologies, Grenoble, France) plus 1/6 complete culture medium (v/v) were plated onto petri dishes in the presence or absence of LPV. Colonies ( $>50$ cells) were scored after 30 days of incubation at $37^{\circ} \mathrm{C}$ in a humidified atmosphere containing $5 \% \mathrm{CO}_{2}$, according to the manufacturer's instructions.

Annexin-V-labeling analysis. Cells were dissociated and allowed to adhere to tissue culture dishes for $24 \mathrm{~h}$. They were then treated with various concentrations of the different Pls for 24 or $48 \mathrm{~h}$ and stained with annexin-V coupled to FITC (Life Technologies SAS, Saint-Aubin, France) and propidium iodide $(50 \mu \mathrm{g} / \mathrm{ml})$. Analysis of annexin-V-propidium iodide staining was performed by fluorescence-activated cell sorting (FACS) using a FACS Calibure (BD Biosciences, Le Pont de Claix, France).

Immunocytochemistry analysis. Cells grown on coverslips were washed with PBS and fixed with Roti-Histofix (Roth, Lauterbourg, France) for $15 \mathrm{~min}$. Fixed cells were incubated in a PBS solution containing Triton X-100: $0.1 \%(\mathrm{v} / \mathrm{v})$ for $20 \mathrm{~min}$, and treated with PBS containing normal goat serum (5\% v/v) for $30 \mathrm{~min}$. Incubation with anti-activated CASP3 antibody (Cell Signaling Technology-Ozyme, Saint-Quentin en Yvelines, France) was carried out at $4{ }^{\circ} \mathrm{C}$ overnight followed by incubation with antibody coupled to a red fluorophore, as indicated in the figure legend and phalloidin coupled to Alexa-Fluor 488 (Invitrogen, Cergy-Pontoise, France) to visualize actin fibers. Primary antibodies were omitted for the negative controls. Nuclei were counterstained with Hoechst 33258 (0.5 $\mu \mathrm{g} / \mathrm{ml}$; Invitrogen). Coverslips were mounted using gel mount before visualization using an Axio observer Z1 microscope (Carl Zeiss Microlmaging, Inc., Thornwood, NY, USA).

Western blot. Cells were rinsed in ice-cold PBS and solubilized in stop buffer $^{48}$ in the presence of Complete PI cocktail (Roche Diagnostics).

Sixty micrograms of proteins were resolved by $8 \%$ (PARP-1 or BiP analysis) or $15 \%$ (CASP3 analysis) SDS-PAGE under reducing conditions and transferred to immobilon-P membranes (Millipore, Molshiem, France). For immunoblotting assays, the detection antibodies were: rabbit anti-total or anti-activated CASP3, mouse antimurine cleaved PARP-1 (Asp 214; Cell Signaling Technology-Ozyme), mouse antiKDEL motif (Stressgen, Tebu-Bio, Le Perray en Yvelines, France) to detect BiP ${ }^{56}$ and mouse anti- $\beta$-tubulin I (Sigma). They were diluted in Tris-buffered saline (pH 7.6) containing $0.1 \%$ Tween 20 and $5 \%$ nonfat dry milk, as indicated in Supplementary Table 1. The membranes were incubated with the diluted antibody overnight at $4{ }^{\circ} \mathrm{C}$.

The bound primary antibody was detected by horseradish peroxidase-conjugated secondary antibody and visualized using an ECL detection kit (Millipore).

Chemiluminescence was observed and quantified using a molecular imager ChemiDoc XRS system (Bio-Rad, Marne la Coquette, France). The band intensity was measured using Bio-Rad Quantity One software.

Effect of LPV on allograft formation. Allograft induction was performed as previously described. ${ }^{14}$ Briefly, single-cell suspensions were prepared in a PBS-Matrigel (BD Bioscience) mixture (v/v) and injected in a $100 \mu \mathrm{l}$ volume 
subcutaneously in the back of CB17/SCID mice (Charles River Laboratories, L'Arbresle, France) anesthetized by i.p. injection of ketamine/xylasine (50 and $10 \mathrm{mg} / \mathrm{kg}$, respectively). Then the mice were divided into two groups: one group received an i.p. injection of a placebo $(200 \mu \mathrm{l}$ of an injectable solution of $5 \%$ glucose $(p / v))$; the other group received a treatment consisting of a twice daily i.p. injection of LPV ( $0.4 \mathrm{mg}$ per mice per injection) and RTV $(0.1 \mathrm{mg}$ per mice per injection) dissolved in $200 \mu \mathrm{l}$ of an injectable solution of $5 \%$ glucose $(\mathrm{p} / \mathrm{v})$.

This dosage was chosen to be in the range of dosages administered to humans and to avoid both toxicity and negative side effects that might develop upon a longterm treatment of mice. ${ }^{37}$

The mice were monitored twice daily for 55 days and then killed by $\mathrm{CO}_{2}$ asphyxiation and their organs were resected and submitted to histological analysis.

Investigations were conducted according to the French and European rules for care and use of research animals. They were carried out under the supervision of certified researchers in accordance with good animal practice, as defined by the French 'Direction des Services Vétérinaires'.

Mouse imaging. Bioluminescent imaging of inoculated cells was performed using a Xenogen IVIS 100 Imaging System (Xenogen Biosciences, Cranbury, NJ, USA). Before imaging, CB17/SCID mice were anesthetized in a chamber with a 1.5\% (v/v) isoflurane/air mixture and injected i.p. with luciferin $(150 \mathrm{mg} / \mathrm{kg}$ body weight).

Validation of the data obtained by bio-imaging to assess the extent of allografts was performed after autopsy of killed mice.

Histological analysis. Histological analysis was performed on tumors fixed in formalin and embedded in paraffin. Sections were stained with hematoxylin, eosin and safran.

Statistical analysis. The results are shown as mean \pm S.E.M. with the number of experiments indicated. Statistical significance was determined by $t$-tests or ANOVA using Micrococal Origin 6.0 (Micrococal Software, Northampton MA, USA). Probability values $<0.05$ were considered statistically significant and are marked with a single asterisk, $<0.01$ with double asterisks and $<0.001$ with triple asterisks

\section{Conflict of Interest}

The authors declare no conflict of interest.

Acknowledgements. We thank our colleagues, for helpful suggestions and discussions. We are indebted to, Drs. Pascal Peraldi and Didier Pisani, for a careful reading of the manuscript. We acknowledge the expert technical assistance of Agnes Loubat with flow cytometry; Franck Paput and Cendrine Dubaud with animal care. Our lab receives financial support from Institut National du Cancer (INCa; grant number: CT 30008187) and from Agence Nationale de Recherche sur le SIDA (ANRS; grant number: AO-2013-1). Pauline Martin is recipient of a predoctoral fellowship from La Ligue Contre le Cancer.

1. Clarke MF, Dick JE, Dirks PB, Eaves CJ, Jamieson CH, Jones DL et al. Cancer stem cellsperspectives on current status and future directions: AACR Workshop on cancer stem cells. Cancer Res 2006; 66: 9339-9344.

2. Sleeman J, Steeg PS. Cancer metastasis as a therapeutic target. Eur J Cancer 2010; 46: $1177-1180$.

3. Shipitsin M, Campbell LL, Argani P, Weremowicz S, Bloushtain-Qimron N, Yao J et al. Molecular definition of breast tumor heterogeneity. Cancer Cell 2007; 11: 259-273.

4. Sugihara E, Saya H. Complexity of cancer stem cells. Int J Cancer 2012; 132: 1249-1259.

5. Valent $\mathrm{P}$, Bonnet D, De Maria R, Lapidot T, Copland M, Melo JV et al. Cancer stem cell definitions and terminology: the devil is in the details. Nat Rev Cancer 2012: 12: 767-775.

6. Al-Hajj M, Wicha MS, Benito-Hernandez A, Morrison SJ, Clarke MF. Prospective identification of tumorigenic breast cancer cells. Proc Natl Acad Sci USA 2003; 100 3983-3988

7. Li C, Heidt DG, Dalerba P, Burant CF, Zhang L, Adsay V et al. Identification of pancreatic cancer stem cells. Cancer Res 2007; 67: 1030-1037.

8. Ricci-Vitiani L, Lombardi DG, Pilozzi E, Biffoni M, Todaro M, Peschle $\mathrm{C}$ et al. Identification and expansion of human colon-cancer-initiating cells. Nature 2007; 445: 111-115.

9. Singh SK, Hawkins C, Clarke ID, Squire JA, Bayani J, Hide T et al. Identification of human brain tumour initiating cells. Nature 2004; 432: 396-401.

10. Ginestier C, Hur MH, Charafe-Jauffret E, Monville F, Dutcher J, Brown M et al. ALDH1 is a marker of normal and malignant human mammary stem cells and a predictor of poor clinical outcome. Cell Stem Cell 2007; 1: 555-567.
11. Kondo T, Setoguchi T, Taga T. Persistence of a small subpopulation of cancer stem-like cells in the C6 glioma cell line. Proc Natl Acad Sci USA 2004; 101: 781-786.

12. Reya T, Morrison SJ, Clarke MF, Weissman IL. Stem cells, cancer, and cancer stem cells. Nature 2001; 414: 105-111.

13. Baccelli I, Trumpp A. The evolving concept of cancer and metastasis stem cells. J Cell Biol 2012; 198: 281-293.

14. Darini CY, Pisani DF, Hofman P, Pedeutour F, Sudaka I, Chomienne C et al. Self-renewal gene tracking to identify tumour-initiating cells associated with metastatic potential. Oncogene 2012; 31: 2438-2449.

15. Ben-Porath I, Thomson MW, Carey VJ, Ge R, Bell GW, Regev A et al. An embryonic stem cell-like gene expression signature in poorly differentiated aggressive human tumors. Nat Genet 2008; 40: 499-507.

16. Chiou SH, Wang ML, Chou YT, Chen CJ, Hong CF, Hsieh WJ et al. Coexpression of Oct4 and Nanog enhances malignancy in lung adenocarcinoma by inducing cancer stem celllike properties and epithelial-mesenchymal transdifferentiation. Cancer Res 2010; 70: 10433-10444.

17. Clement V, Sanchez P, de Tribolet N, Radovanovic I, Ruiz i Altaba A. HEDGEHOG-GLI1 signaling regulates human glioma growth, cancer stem cell self-renewal, and tumorigenicity. Curr Biol 2007; 17: 165-172.

18. Bao S, Wu Q, McLendon RE, Hao Y, Shi Q, Hjelmeland AB et al. Glioma stem cells promote radioresistance by preferential activation of the DNA damage response. Nature 2006; 444: 756-760.

19. Dean M. Cancer stem cells: implications for cancer causation and therapy resistance. Discov Med 2005; 5: 278-282.

20. Diehn M, Cho RW, Clarke MF. Therapeutic implications of the cancer stem cell hypothesis. Semin Radiat Oncol 2009; 19: 78-86.

21. Diehn M, Clarke MF. Cancer stem cells and radiotherapy: new insights into tumor radioresistance. J Natl Cancer Inst 2006; 98: 1755-1757.

22. Eyler CE, Rich JN. Survival of the fittest: cancer stem cells in therapeutic resistance and angiogenesis. J Clin Oncol 2008; 26: 2839-2845.

23. Woodward WA, Chen MS, Behbod F, Alfaro MP, Buchholz TA, Rosen JM. WNT/betacatenin mediates radiation resistance of mouse mammary progenitor cells. Proc Natl Acad Sci USA 2007; 104: 618-623.

24. Gavrilov K, Saltzman WM. Therapeutic siRNA: principles, challenges, and strategies. Yale J Biol Med 2012; 85: 187-200.

25. Jin L, Hope KJ, Zhai Q, Smadja-Joffe F, Dick JE. Targeting of CD44 eradicates human acute myeloid leukemic stem cells. Nat Med 2006; 12: 1167-1174.

26. Marangoni E, Lecomte N, Durand L, de Pinieux G, Decaudin D, Chomienne C et al. CD44 targeting reduces tumour growth and prevents post-chemotherapy relapse of human breast cancers xenografts. Br J Cancer 2009; 100: 918-922.

27. Autran B, Carcelaint G, Li TS, Gorochov G, Blanc C, Renaud M et al. Restoration of the immune system with anti-retroviral therapy. Immunol Lett 1999; 66: 207-211.

28. Vella S, Palmisano L. Antiretroviral therapy: state of the HAART. Antiviral Res 2000; 45: 1-7.

29. Gill J, Bourboulia D, Wilkinson J, Hayes P, Cope A, Marcelin AG et al. Prospective study of the effects of antiretroviral therapy on Kaposi sarcoma-associated herpesvirus infection in patients with and without Kaposi sarcoma. J Acquir Immune Defic Syndr 2002; 31: 384-390.

30. Monini P, Sgadari C, Grosso MG, Bellino S, Di Biagio A, Toschi E et al. Clinical course of classic Kaposi's sarcoma in HIV-negative patients treated with the HIV protease inhibitor indinavir. Aids 2009; 23: 534-538.

31. McLean K, VanDeVen NA, Sorenson DR, Daudi S, Liu JR. The HIV protease inhibitor saquinavir induces endoplasmic reticulum stress, autophagy, and apoptosis in ovarian cancer cells. Gynecol Oncol 2009; 112: 623-630.

32. Pyrko P, Kardosh A, Wang W, Xiong W, Schonthal AH, Chen TC. HIV-1 protease inhibitors nelfinavir and atazanavir induce malignant glioma death by triggering endoplasmic reticulum stress. Cancer Res 2007; 67: 10920-10928.

33. Yang Y, Ikezoe T, Takeuchi T, Adachi Y, Ohtsuki Y, Takeuchi S et al. HIV-1 protease inhibitor induces growth arrest and apoptosis of human prostate cancer $\mathrm{LNCaP}$ cells in vitro and in vivo in conjunction with blockade of androgen receptor STAT3 and AKT signaling. Cancer Sci 2005; 96: 425-433.

34. Esposito V, Palescandolo E, Spugnini EP, Montesarchio V, De Luca A, Cardillo I et al. Evaluation of antitumoral properties of the protease inhibitor indinavir in a murine model of hepatocarcinoma. Clin Cancer Res 2006; 12: 2634-2639.

35. Chaitanya GV, Steven AJ, Babu PP. PARP-1 cleavage fragments: signatures of cell-death proteases in neurodegeneration. Cell Commun Signal 2010; 8: 31.

36. Gupta PB, Onder $\Pi$, Jiang G, Tao K, Kupenwasser C, Weinberg RA et al, Identification of selective inhibitors of cancer stem cells by high-throughput screening. Cell 2009; 138: 645-659.

37. Prot M, Heripret L, Cardot-Leccia N, Perrin C, Aouadi M, Lavrut T et al. Long-term treatment with lopinavir-ritonavir induces a reduction in peripheral adipose depots in mice. Antimicrob Agents Chemother 2006; 50: 3998-4004

38. Magee JA, Piskounova E, Morrison SJ. Cancer stem cells: impact, heterogeneity, and uncertainty. Cancer Cell 2012; 21: 283-296.

39. Monini P, Sgadari C, Toschi E, Barillari G, Ensoli B. Antitumour effects of antiretroviral therapy. Nat Rev Cancer 2004; 4: 861-875.

40. Ikezoe T, Saito T, Bandobashi K, Yang Y, Koeffler HP, Taguchi H. HIV-1 protease inhibitor induces growth arrest and apoptosis of human multiple myeloma cells via inactivation of signal transducer and activator of transcription 3 and extracellular signal-regulated kinase 1/2. Mol Cancer Ther 2004; 3: 473-479. 
41. Gills JJ, Lopiccolo J, Tsurutani J, Shoemaker RH, Best CJ, Abu-Asab MS et al. Nelfinavir, a lead HIV protease inhibitor, is a broad-spectrum, anticancer agent that induces endoplasmic reticulum stress, autophagy, and apoptosis in vitro and in vivo. Clin Cancer Res 2007; 13: 5183-5194.

42. Johnson MD, O'Connell M, Pilcher W. Lopinavir inhibits meningioma cell proliferation by Akt independent mechanism. J Neurooncol 2011; 101: 441-448.

43. Sachlos E, Risueno RM, Laronde S, Shapovalova Z, Lee JH, Russell J et al. Identification of drugs including a dopamine receptor antagonist that selectively target cancer stem cells. Cell 2012; 149: 1284-1297.

44. Dash C, Kulkarni A, Dunn B, Rao M. Aspartic peptidase inhibitors: implications in drug development. Crit Rev Biochem Mol Biol 2003; 38: 89-119.

45. Benes $P$, Vetvicka V, Fusek M. Cathepsin D-many functions of one aspartic protease. Crit Rev Oncol Hematol 2008; 68: 12-28.

46. Sham HL, Zhao C, Li L, Betebenner DA, Saldivar A, Vasavanonda S et al. Novel lopinavir analogues incorporating non-aromatic P-1 side chains-synthesis and structure-activity relationships. Bioorg Med Chem Lett 2002; 12: 3101-3103.

47. Sham HL, Kempf DJ, Molla A, Marsh KC, Kumar GN, Chen CM et al. ABT-378, a highly potent inhibitor of the human immunodeficiency virus protease. Antimicrob Agents Chemother 1998; 42: 3218-3224.

48. Djedaini M, Peraldi P, Drici MD, Darini C, Saint-Marc P, Dani C et al. Lopinavir co-induces insulin resistance and ER stress in human adipocytes. Biochem Biophys Res Commun 2009; 386: 96-100.

49. Caron-Debarle M, Boccara F, Lagathu C, Antoine B, Cervera P, Bastard JP et al. Adipose tissue as a target of HIV-1 antiretroviral drugs. Potential consequences on metabolic regulations. Curr Pharm Des 2010; 16: 3352-3360.

50. Vernochet C, Azoulay S, Duval D, Guedj R, Cottrez F, Vidal H et al. Human immunodeficiency virus protease inhibitors accumulate into cultured human adipocytes and alter expression of adipocytokines. J Biol Chem 2005; 280: 2238-2243.
51. Caron M, Auclair M, Lagathu C, Lombes A, Walker UA, Kornprobst M et al. The HIV-1 nucleoside reverse transcriptase inhibitors stavudine and zidovudine alter adipocyte functions in vitro. Aids 2004; 18: 2127-2136.

52. Zhang S, Carper MJ, Lei X, Cade WT, Yarasheski KE, Ramanadham S. Protease inhibitors used in the treatment of HIV + induce beta-cell apoptosis via the mitochondrial pathway and compromise insulin secretion. Am J Physiol Endocrinol Metab 2009; 296 E925-E935.

53. Stoner EJ, Cooper AJ, Dickman DA, Kolaczkowski L, Lallaman JE, Liu JH et al. Synthesis of HIV Protease Inhibitor ABT-378 (Lopinavir). Org Process Res Dev 2000; 4 264-269.

54. Wdziekonski B, Villageois P, Dani C. Development of adipocytes from differentiated ES cells. Methods Enzymol 2003; 365: 268-277.

55. Rodriguez AM, Pisani D, Dechesne CA, Turc-Carel C, Kurzenne JY, Wdziekonski B et al. Transplantation of a multipotent cell population from human adipose tissue induces dystrophin expression in the immunocompetent mdx mouse. J Exp Med 2005; 201 1397-1405.

56. Thuerauf DJ, Marcinko M, Gude N, Rubio M, Sussman MA, Glembotski CC. Activation of the unfolded protein response in infarcted mouse heart and hypoxic cultured cardiac myocytes. Circ Res 2006; 99: 275-282.

(i) Cell Death and Disease is an open-access journal published by Nature Publishing Group. This work is licensed under a Creative Commons Attribution 3.0 Unported License. To view a copy of this license, visit http://creativecommons.org/ licenses/by/3.0/

\section{Supplementary Information accompanies this paper on Cell Death and Disease website (http://www.nature.com/cddis)}

